\title{
APPLICATION OF IFSAR TECHNOLOGY IN TOPOGRAPHIC MAPPING: JUPEM'S EXPERIENCE
}

\author{
Sr Ahamad Zakaria ${ }^{a}$ \\ ${ }^{a}$ Department of Survey and Mapping Malaysia (JUPEM), Policy and Mapping Coordination Division, Jalan Sultan Yahya Petra \\ 50578 Kuala Lumpur, email: ahamad.zakaria@jupem.gov.my
}

\begin{abstract}
The application of Interferometric Synthetic Aperture Radar (IFSAR) in topographic mapping has increased during the past decades. This is due to the advantages that IFSAR technology offers in solving data acquisition problems in tropical regions. Unlike aerial photography, radar technology offers wave penetration through cloud cover, fog and haze. As a consequence, images can be made free of any natural phenomenon defects. In Malaysia, Department of Survey and Mapping Malaysia (JUPEM) has been utilizing the IFSAR products since 2009 to update topographic maps at 1:50,000 map scales. Orthorectified radar imagery (ORI), Digital Surface Models (DSM) and Digital Terrain Models (DTM) procured under the project have been further processed before the products are ingested into a revamped mapping workflow consisting of stereo and mono digitizing processes. The paper will highlight the experience of Department of Survey and Mapping Malaysia (DSMM)/ JUPEM in using such technology in order to speed up mapping production.
\end{abstract}

Keywords: Interferometric Synthetic Aperture Radar (IFSAR), Orthorectified Radar Imagery (ORI), Digital Surface Models (DSM) and Digital Terrain Models (DTM).

\section{Introduction}

Department of Survey and Mapping Malaysia (JUPEM) have been using various kinds of data sources for their topographic mapping exercise. The main source of data has been aerial photographs taken by using conventional analogue camera, RC30 or by the newly acquired digital camera, DMC. Being optical sensor, both cameras which are fully dependent on the availability of the Sun's radiation for their operations can only image area not covered by cloud. In tropical region where cloud cover has become a perennial problem, these shortcomings have become the main obstacle for JUPEM to deliver the mapping services. Among the affected areas are the hinterland of Sarawak and Sabah and also the central part of Peninsular Malaysia. JUPEM, being the sole agency responsible for topographic mapping in the country have been trying using other forms of satellite imageries such as Ikonos, Quickbird and World View 1, but to no avail. Despite knowing that all the sensors are optical sensors, JUPEM were hopeful that by having higher frequencies of imaging by many sensors could rectify the problems. However, it was soon found that it was only a futile effort. With regards to alternative solutions, JUPEM have decided to embark on using the Synthetic Aperture Radar (SAR) technology for the problematic area. This is not done in ad-hoc manner but after careful considerations on the pros and cons of the technology. Concurrently, JUPEM have been doing a lot of intense research by continuously sending staffs for IFSAR courses overseas as well as through technology update sessions given by IFSAR experts from various related technology providers. As a result, a pilot project was initiated in 2009 with two project areas at Bersia (FJ21) in Perak and $\mathrm{Kg}$ Kubang Palas (FJ33) in Kedah for the production of
1:50,000 topographic maps. A year later, more IFSAR datasets have been acquired by JUPEM to satisfy the needs to complete the revision before 2014. The areas include southern Johor, and the hinterland of Sabah and Sarawak. The paper will highlight JUPEM's experience in using such data, the pros and the cons.

\section{Interferometric Synthetic Aperture Radar (IFSAR)}

Interferometric Synthetic Aperture Radar (IFSAR) technology is fast becoming an alternative solution for many mapping agencies around the world. Introduced nearly 40 years ago by Graham [1, pp24], IFSAR technology (also popularly known as InSAR in Europe) is operated on either aircraft platform (airborne IFSAR) or satellite platform (Spaceborne IFSAR). Currently there are two main providers for airborne IFSAR that is Intermap and Fugro while EADS (TerraSAR-X) and MDA (Radarsat 1 and Radarsat 2) are the two main players for space borne IFSAR. Unlike optical sensor which depends on the Sun's radiation to operate, IFSAR technology employs active sensor which transmits its own radar signal and receive the echoes or backscatter from the imaged objects. The typical operational wavelengths are X-band and P-band depending on the system mechanics used. For example Intermap use only $\mathrm{X}$-band while Fugro utilize both radar bands i.e. X-band and P-band together by using much bigger aircraft.

One of the main characteristics of radar signal is that different bands would give different penetration of the canopies. For example, transmission of X-band signals can only reach the surface of the canopy and will be reflected back to the radar sensor (Figure 1-left). P-band signals with longer wavelengths, on the other hand, can penetrate more into the canopies and experience 
volumetric scattering before finally reflected back to the sensor (Figure 1-right). The delay in time of transmission and return of signals will determine different depths of the imaged terrain. The behaviour of the return signals (known as backscatter) also indicates the properties of the terrain imaged. Low reflectance signifies smooth surface such as water body and road networks. Intense returns of signals for example shows rough surface on the ground including town area or hilly area facing the radar sensor [4].

\section{IFSAR Products}

Since the IFSAR data was procured from Intermap, what follows are a quick overview of the core products specifications namely Orthorectified Radar Images (ORIs), Digital Surface Models (DSMs) and Digital Terrain Models (DTMs).

\begin{tabular}{|l|c|l|c|l|l|l|}
\hline $\begin{array}{l}\text { Acquisitio } \\
n\end{array}$ & \multicolumn{2}{|c|}{ ORI } & \multicolumn{2}{|c|}{ DSM } & \multicolumn{2}{c|}{ DTM } \\
\cline { 2 - 6 } Accuracy & $\begin{array}{l}\text { Pixel } \\
\text { size } \\
(\mathrm{m})\end{array}$ & $\begin{array}{l}\text { Planime } \\
\text { tric } \\
\text { RMSE } \\
(\mathrm{m})\end{array}$ & $\begin{array}{l}\text { Posting } \\
(\mathrm{m})\end{array}$ & $\begin{array}{l}\text { Vertical } \\
\text { RMSE } \\
(\mathrm{m})\end{array}$ & $\begin{array}{l}\text { Postin } \\
\mathrm{g}(\mathrm{m})\end{array}$ & $\begin{array}{l}\text { Vertical } \\
\text { RMSE } \\
(\mathrm{m})\end{array}$ \\
\hline $\begin{array}{l}\text { IFSAR } \\
\text { Type I }\end{array}$ & 0.62 & 2 & 5 & 0.7 & 5 & 0.7 \\
\hline
\end{tabular}

Table 1: Product specification for IFSAR Core Products from Intermap (Source: Intermap [2])

Intermap products were captured by using X-band signals and thus could not penetrate the canopies. Therefore no automatic DTM can be captured at first instance. The DSM has been processed further by Intermap in order to extract the DTM. The ORI however is more resourceful and extensive since the resolution is less than 1 meter ( 0.625 meter). The detail specification of Intermap's IFSAR products is as shown in Table 1.

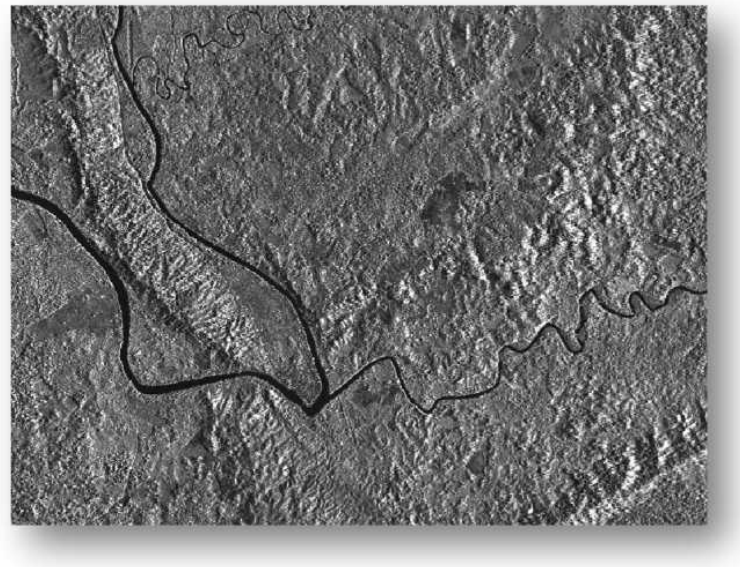

Figure 1(a): X-band (Source: Fugro [3])

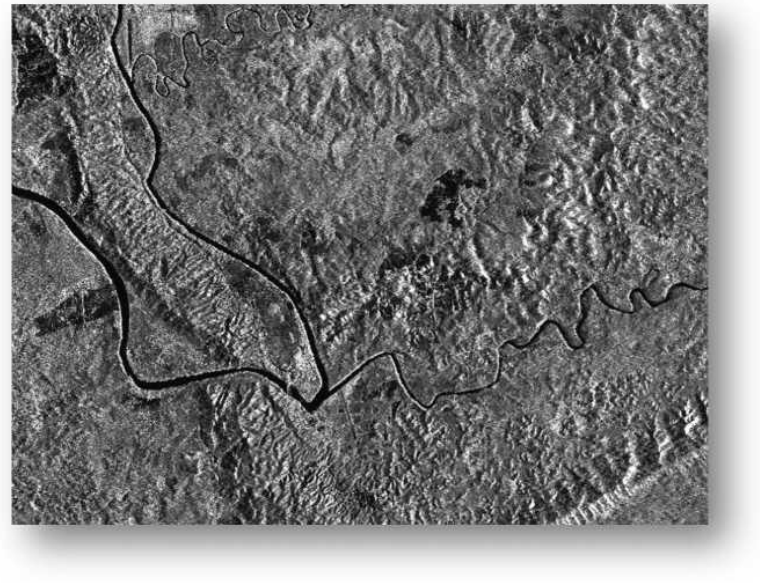

Figure 1(b): P-band (Source: Fugro [3])

The ORI look somewhat like monochromatic aerial photos and are always generated in pairs to allow single pass interferometry technology to work. Consequently, interferometric products such as interferograms and unwrapped phase image can be generated. This leads to the DSM subsequently derived. As DSMs display the first surface on the ground that the radar strikes, they therefore include all terrain features such as buildings, power lines, and treed areas. Before the DSMs can be used for topographic mapping, all features must first be removed to create DTM in the form of contour lines [1].

\subsection{Orthorectified Radar Image (ORI)}

An ORI is a grayscale cloud free image of the Earth's surface that has been corrected to remove geometrical distortions. The key feature of the product is that it provides a means of viewing the earth's surface in a way that accentuates features far more than is possible with aerial photography. The radar sensor looks to the side of the aircraft and casts "shadows" that enable users to visually perceive the elevation information in the image, even if they are unfamiliar with the underlying technology. High resolution ORIs are ideally suited for interpretation and feature extraction, enabling more accurate topographic line mapping and GIS data compilation. Figure 2 is an example of an ORI from Intermap's NEXTMap Britain project, which involved mapping all of Great Britain [1]. 


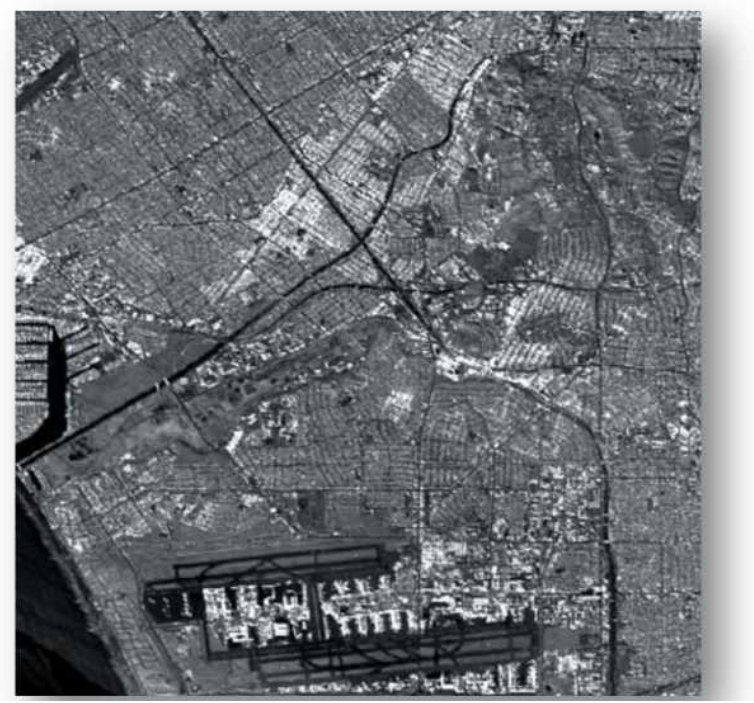

Figure 2: ORI example (Intermap [1])

\subsection{Digital Surface Model (DSM)}

A DSM is a topographic model of the earth's surface comprised of elevation measurements that are laid out on a grid. These measurements are derived from the return signals received by the two radar antennae on the aircraft. A DSM is the first surface the signals bounce of any object on the ground that could include buildings, roads, vegetation, as well as other natural terrain

features. The key feature of this product is that it provides a geometrically correct reference frame for georeferencing of other data layers. For example, DSM can be used to create 3D fly- through, augment simulated environments, and conduct view-shed analyses. Figure 3left shows the corresponding DSM for the same region displayed in Figure 2. In this color map, blue has been associated with the lowest elevation, and red with the highest [1].

\subsection{Digital Terrain Model (DTM)}

A DTM is a topographic model of the bare earth which has had vegetation, buildings and other cultural features digitally removed by means of specialized software, leaving just the underlying terrain. Sometimes referred to as bare earth model, DTM enables users to infer terrain characteristics possibly hidden in the DSM. DTM supports many applications including topographic maps in the form of contour lines, the base for flood plain analyses, agricultural, and intelligent vehicle applications. In Figure 3-right, we can see how the buildings that were evident in the previous figures are no longer visible [1].
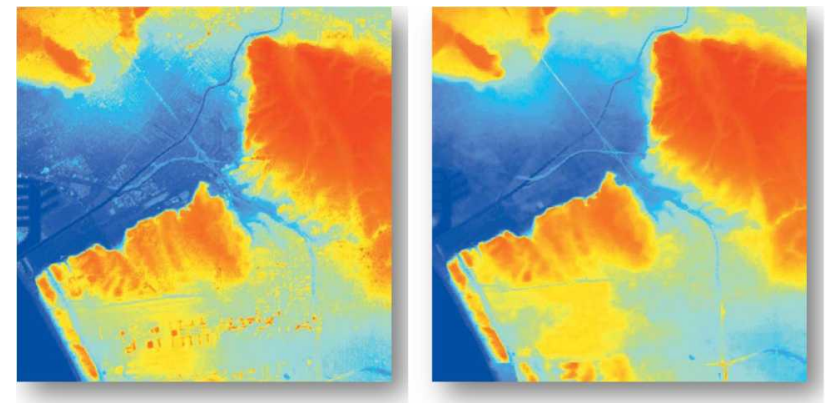

Figure 3: Examples of DSM - left and DTM - right (Intermap [1])

\section{Topographic Data Capture using IFSAR data}

The availability of these new sources of data has given a lot of options on how topographic features can be extracted. The concept of mono and stereo-digitizing based on aerial photographs has now been utilized for IFSAR data. Upon receiving the data, there are a number of steps that has to be performed before operators can start the compilation work either in stereo or mono mode. Firstly, the ORIs have to be reprojected to the Malaysian Coordinate System (GDM2000) since the default format is WGS84 Coordinate System. This is important to ensure that no planimetric errors are made during digitizing process. Once the ORIs are converted into GDM2000 coordinate system and loaded into the CAD system, the process of digitizing in mono mode can promptly begin.

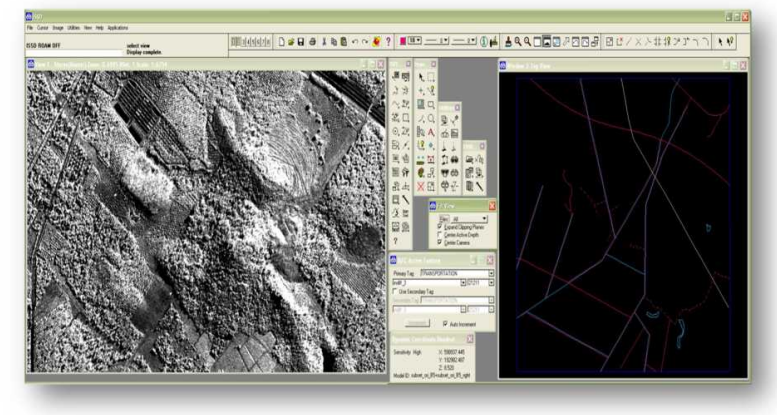

Figure 4: 3D compilation process from IFSAR stereo mate pair by using Intergraph Image Station Stereo Display (ISSD)

But for stereo digitizing process (Figure 4), there are more steps involved. By processing the ORI and DSM in the photogrammetric software, a stereo-mate for the ORI must first be generated. Subsequently, the ORI and its stereo-mate can then be loaded as stereo-pair in stereodigitizing display unit for data extraction tasks. Typical layers used in topographic mapping specification for 1:50,000 MY501T series includes buildings, vegetation, transportation and utilities. Simultaneously, the work on hypsography layers can also continue in parallel. By further processing the DTM by using Global Mapper software, contour lines and spot heights are produced. Some editing of the contour lines is possibly required for cartographic quality.

Undeniably, the utilization of IFSAR requires a thorough understanding of SAR image itself. For that matter, two specialized courses have been conducted on SAR image interpretation; mono (2D) and stereo (3D) interpretations and map compilations. During the course, a new workflow has been designed to suit the capabilities of the existing photogrammetric software in the Data Acquisition Section. Examples of IFSAR DTM and the derived contour lines are shown in Figure 5. 

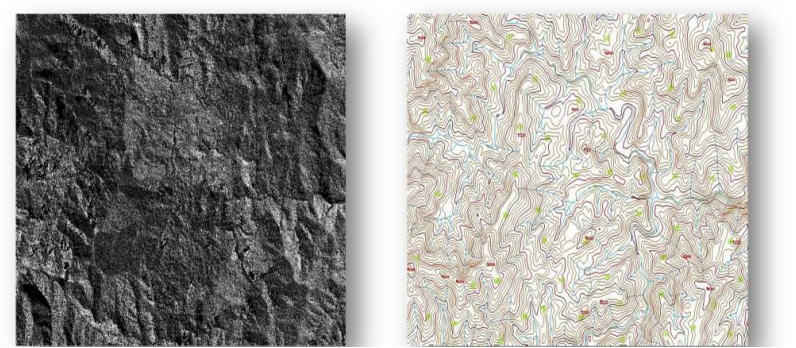

Fig 5: DTM from IFSAR (left) and contour lines derived from DTMIFSAR (right) of Bersia in Perak

\section{The Advantages and Disadvantages of using IFSAR data}

After receiving these products, the workflow to produce topographic maps had become much simpler. Once the coordinate reference of the ORI is converted to GDM2000, the planimetric data extraction can be started. Parallel to that, by using Global Mapper software, contour lines layers at 20 meters interval required for the MY501T map sheet series can be automatically generated from the DTM. Although the steps are purely automatic, only minimal editing are needed to satisfy the cartographic map quality standard. Previously, the generation of contour lines is performed by using stereo photogrammetric method which is a time consuming process even in digital photogrammetric environment. The project has also shown that the problematic areas perennially under cloud cover that entail dense forests such as Bersia (FJ21) in Perak and Kg Kubang Palas (FJ33) in Kedah and densely vegetated area of Johor (BF44) can be mapped at according to the map specification of 1:50,000 scale.

Nevertheless, there were also a lot of problems that cropped up during the mapping exercise. Since radar image is black and white with the natural artifacts such as layover, foreshortening and layovers, at initial stage, the operators were having a tough time to classify the features. For example, when buildings features are surrounded by trees especially in villages, they were able to identify only 3 out of 10 houses. The operators have also become confused in classifying playing ground and buildings since the image is only in black and white. There are features that can be easily classified such as oil palm, rubber and dense forest. It was also very difficult to categorize road classes such as between dirt road and tarred road. As for utility features, only transmission lines can be recognized since the reserve buffer stretch is strongly visible. During the project, it was also found that different types of vegetations were digitized wrongly. If taken for granted, the scenario could increase the burden of field work in clarifying the information on the ground.

Although there are a lot of problems faced by the operators in the identification of features, extra measures have been identified and proposed to alleviate or reduce the problems. Besides using IFSAR stereomate method, the establishment of cross-referencing procedures with existing vector data and maps can also be very helpful. The usage of other optical images such as Ikonos,
Quickbird and Google Earth for the problematic area is also of paramount importance. The procedures of highlighting the ambiguous area in the datasets for easy checking by Topographic Section should also be established.

\section{Conclusions}

From the experience of capturing topographic features into vector form for the three map sheets namely FJ33, FJ21 and BF44, it can be concluded that the usage of IFSAR data in the map compilation program is suitable for medium scale maps that is for 1:50,000 scale. For large scale mapping where many features of different sizes and shapes must be digitized to meet the specification, IFSAR products are not sufficient even with stereo environment workflow. This is as a result of the image properties of the radar itself. Secondary data sources such as IKONOS, Quickbird must be made available beforehand to assist the map compilation. If there is no optical image available, the last alternative would be to leave the updating process for field work completion steps. In terms of vertical requirements, the derived contour lines from DTM are considered suitable for medium scale or even for large scale mapping since the required contour intervals are only 5 meters compared to IFSAR data which has higher vertical accuracy.

Unmistakably, the application of IFSAR in JUPEM's mapping workflow requires a paradigm shift in the approaches previously undertaken when aerial photographs are the only data source used for map production. Although the current radar technology is not able to match the same accuracy as the aerial photogrammetry, IFSAR also has many advantages when rapid medium scale mapping is warranted. If the planning is exercised diligently, the usage of IFSAR/SAR products not only can complement the existing aerial photogrammetric method but also facilitate JUPEM to achieve the objectives.

\section{References}

Intermap_ProdHandbook_v4.3.pdf

www.pcigeomatics.com/support/pdf/Intermap

ProdHandbook v4.3.pdf

Mapping Services - IFSAR-based Products: http://www.intermap.com/en-

us/geospatialservicesservices/mappingservices.aspx

Fugro official website: http:/www.geosar.com/ downloads.php

Che Cob, A.S. (2002), An Investigation into Interferometric SAR Processing with Special Reference to Tropical Rain Forest, MSc Thesis, Stuttgart University of Applied Sciences 